\title{
Prognostic value of monocarboxylate transporter 4 in patients with esophageal squamous cell carcinoma
}

\author{
BO CHENG ${ }^{1}$, XUAN $\mathrm{CHEN}^{2}$, YANKANG $\mathrm{LI}^{1}$, XIAOCHEN HUANG ${ }^{2}$ and JINMING YU ${ }^{1}$ \\ ${ }^{1}$ Department of Radiation Oncology, Shandong Cancer Hospital Affiliated to Shandong University, \\ Jinan, Shandong 250117; ${ }^{2}$ Department of Radiation Oncology, Qilu Hospital of \\ Shandong University, Jinan, Shandong 250012, P.R. China
}

Received April 11, 2018; Accepted September 7, 2018

DOI: $10.3892 /$ or.2018.6706

\begin{abstract}
Monocarboxylate transporter 4 (MCT4) is a membrane transporter of monocarboxylates that has been reported to play an important role in tumorigenesis and progression in several solid tumor types. The present study aimed to investigate its clinical significance in esophageal squamous cell carcinoma (ESCC). After obtaining and analyzing MCT4 mRNA expression data from The Cancer Genome Atlas (TCGA) database, the prognostic potential of MCT4 was evaluated by IHC analysis. The effect of the knockdown of MCT4 by shRNA was also evaluated using Cell Counting Kit-8 (CCK-8) and clonogenic assays, in order to determine whether MCT4 inhibition affected the proliferation and survival ability of ESCC cells. Flow cytometric analysis was used to evaluate apoptosis. Western blot analysis was performed to detect the expression levels of p-Akt, Bax, Bcl-2, cytoplasmic cytochrome $c$ and cleaved caspase-3. MCT4 expression was associated with $\mathrm{T}$ stage $(\mathrm{P}=0.001), \mathrm{N}$ stage $(\mathrm{P}=0.020)$ and formalin-fixed and paraffin-embedded (TNM) stage $(\mathrm{P}=0.042)$. Kaplan-Meier survival analysis indicated that patients in the high-MCT4 group had a lower overall survival (OS) rate $(\mathrm{P}=0.001)$ and progression-free survival (PFS) rate $(\mathrm{P}=0.003)$. The univariate Cox regression analysis and multivariate Cox regression analysis results indicated that MCT4 is an independent predictor of OS $(\mathrm{P}=0.001$ and 0.014$)$ and PFS ( $\mathrm{P}=0.004$ and 0.046). Downregulation of MCT4 inhibited cell proliferation and increased apoptosis in vitro. The proliferation rate and clone numbers were decreased and apoptotic rates were increased in the sh-MCT4 groups (all $\mathrm{P}<0.05)$. Furthermore, MCT4 knockdown reduced the activation of Akt and increased Bax/Bcl-2 ratios, cytochrome $c$ release and
\end{abstract}

Correspondence to: Dr Jinming Yu, Department of Radiation Oncology, Shandong Cancer Hospital Affiliated to Shandong University, 440 Jiyan Road, Jinan, Shandong 250117, P.R. China E-mail: sdujinmingyu@126.com

Key words: monocarboxylate transporter 4, esophageal squamous cell carcinoma, prognosis, cell proliferation, apoptosis caspase-3 cleavage (all $\mathrm{P}<0.05$ ). Consequently, MCT4 could serve as a promising biomarker for ESCC to identify patients with poor prognosis.

\section{Introduction}

Esophageal carcinoma is the sixth leading cause of cancerrelated mortality worldwide (1), and $90 \%$ of the 456,000 annual new cases of this cancer are esophageal squamous cell carcinoma (ESCC) (2). Such as other malignancies, the most common biological changes in the pathogenesis of ESCC include activation of oncogenes and inactivation of tumorsuppressor genes (3). Considerable effort has been made to identify molecular markers related to the etiology of ESCC (4). However, unlike the estrogen and progesterone receptors in breast cancer, and $\alpha$-fetoprotein (AFP) in hepatocellular carcinoma, promising biomarkers with clinical significance for ESCC have remained elusive.

The monocarboxylate transporter (MCT) family, including MCT1, MCT2, MCT3 and MCT4 isoforms, are essential for fast transport of monocarboxylates in mammals, such as the transport of pyruvate, lactate and ketone bodies across the plasma membrane (5). MCT1 and MCT2 are predominantly involved in lactate uptake, thus deriving cellular energy from initiating oxidative phosphorylation of lactate, whereas MCT4 is involved in lactate excretion (6). MCT4 is highly expressed in tissues that rely on glycolysis, such as skeletal muscle fibers and glial cells in the cerebellum (7-9). Upregulated glycolysis with production of lactate and downregulated mitochondrial oxidative phosphorylation metabolism (OXPHOS) commonly exist in cancer (10). Expression of MCT4 in cancer or stromal cells in the tumor microenvironment varies among different types of cancer. Overexpression of MCT4 in both epithelial and stromal cells of breast cancer (11-13), hepatocellular cancer cells $(14,15)$ and pancreatic cancer cells (16) predicted worse outcomes for patients. Furthermore, high expression of MCT4 in stromal cells of colorectal cancer (17), oral squamous cell carcinoma (18) and gastric cancer (19) was revealed to be associated with poor prognosis.

Currently, an association between MCT4 and ESCC prognosis is yet to be demonstrated. In the present study, we investigated whether MCT4 expression may have prognostic value for ESCC patients. Additionally, we used in vitro assays 
to verify its proliferation-inhibiting and apoptosis-promoting effects on ESCC cells.

\section{Materials and methods}

Analysis of mRNA expression using The Cancer Genome Atlas (TCGA) database. The datasets of RNA sequencing information of $81 \mathrm{ESCC}$ tissues and 11 normal tissues were acquired from the The Cancer Genome Atlas (TCGA, https://tcga-data. nci.nih.gov/) database. mRNA expression data of MCT4 were retrieved from these datasets.

Clinical samples and cell lines. Cancer tissues and corresponding adjacent non-tumor tissues were obtained from 110 ESCC patients, who had not received neoadjuvant therapy and were admitted to Qilu Hospital of Shandong University (Jinan, China) from January 2010 to December 2011. The research proposal was approved by the Ethics Committee of Qilu Hospital. Follow-up data of the 110 patients was available for at least 5 years for review. All ESCC samples were confirmed by pathological evaluation. The tumor, node, metastasis (TNM) staging was classified according to the American Joint Committee on Cancer Cancer Staging Manual (7th edition). Other clinicopathological variables of the patients were obtained from clinical and pathological records.

The human ESCC cell lines KYSE150 and Eca109 were purchased from the China Center for Type Culture Collection in 2017 and authenticated by short tandem repeat analysis. Cell lines were routinely cultured at $37^{\circ} \mathrm{C}$ in RPMI-1640 medium (Gibco; Thermo Fisher Scientific, Inc., Waltham, MA, USA) supplemented with $10 \%$ fetal bovine serum (FBS; Gibco; Thermo Fisher Scientific, Inc.) in an incubator with $5 \% \mathrm{CO}_{2}$.

Immunohistochemical (IHC) analysis. Paraffin-embedded tissue sections were dewaxed and gradually rehydrated. After antigen retrieval by microwave irradiation, endogenous peroxidase activity was blocked by incubation with $3 \%$ hydrogen peroxide. Sections were then incubated with $10 \%$ goat serum at $37^{\circ} \mathrm{C}$ for $30 \mathrm{~min}$, followed by incubation with a rabbit anti-MCT4 polyclonal antibody (dilution 1:100; cat. no. 22787-1-AP; ProteinTech Group, Inc., Chicago, IL, USA) overnight at $4^{\circ} \mathrm{C}$. Sections of the negative control were incubated with PBS instead of the primary antibody. Complexes of streptavidin-peroxidase and biotinylated secondary antibody (dilution 1:200; cat. no. SP-9001; Zhongshan Golden Bridge Biotechnology, Co., Ltd., Beijing, China) were added to the sections, followed by incubation at $37^{\circ} \mathrm{C}$ for $30 \mathrm{~min}$. Subsequently, the MCT4 protein was detected by a chromogenic reaction with 3,3'-diaminobenzidine and counterstained with hematoxylin. The expression level of MCT4 was determined by multiplying the staining density value by the number of positive cells. All slides were independently evaluated by two pathologists using a double-blind method. The staining intensity was graded as 0 (no staining), 1 (weak), 2 (moderate) or 3 (intense). The number of positive cells was scored as 0 $(<5 \%), 1(5-25 \%), 2(26-50 \%), 3(51-75 \%)$ or $4(>75 \%)$. The level of expression was defined as 'low expression' if the multiplied score was $<8$; otherwise it was defined as 'high expression'.
Transfection. Plasmids containing shRNA targeting MCT4 (cat. no. HSH022117-LVmRU6GP) and a control shRNA (cat. no. CSHCTR001-LVR- U6GP) were purchased from GeneCopoeia, Inc. (Rockville, MD, USA). When cells reached $80 \%$ confluence, the plasmids were transfected into the KYSE150 and Eca109 cells using Lipofectamine 2000 (Invitrogen; Thermo Fisher Scientific, Inc.). The transfection procedure was carried out according to the manufacturer's protocol. Knockdown efficiency was determined by quantitative real-time polymerase chain reaction (RT-qPCR) and western blot analysis $48 \mathrm{~h}$ after transfection.

RNA isolation and RT-qPCR. Total RNA was isolated from ESCC cells using TRIzol reagent (Invitrogen; Thermo Fisher Scientific, Inc.). Briefly, cells were lysed directly by adding $1 \mathrm{ml}$ of TRIzol to each well of a 6-well plate for $10 \mathrm{~min}$, followed by adding $0.2 \mathrm{ml}$ of chloroform per $1 \mathrm{ml}$ of TRIzol. After shaking tubes vigorously by hand for $30 \mathrm{sec}$ and incubating samples at room temperature for $5 \mathrm{~min}$, the samples were centrifuged at $12,000 \mathrm{x} \mathrm{g}$ for $15 \mathrm{~min}$ at $4^{\circ} \mathrm{C}$. Following centrifugation, RNA remained exclusively in the aqueous phase. Then, the RNA was precipitated from the aqueous phase by mixing with isopropyl alcohol. Finally, the RNA pellet was washed once with $75 \%$ ethanol and dissolved in RNase-free water after airdrying. RT-qPCR was conducted on a Bio-Rad Single Color Real-Time PCR system (Bio-Rad Laboratories, Inc., Hercules, CA, USA), using SYBR-Green Real-Time PCR Master Mix (Toyobo Life Science, Osaka, Japan). The primers used were as follows: MCT4 forward, 5'-TCGTCATCACYGGCTTCTCC-3' and reverse, 5'-ATCCAGGCTGTGTCGCTGTA-3'; $\beta$-actin forward, 5'-CAAAGGCCAACAGAGAGAAGAT-3', and reverse, 5'-TGAGACACACCATCACCAGAAT-3'.

Western blot analysis. Protein was extracted $48 \mathrm{~h}$ after transfection for MCT4 analysis. Other analyses were performed $120 \mathrm{~h}$ after transfection. Total protein was extracted using RIPA lysis buffer (Beyotime Institute of Biotechnology, Shanghai, China) with $1 \%$ phenylmethylsulfonyl fluoride (PMSF) (Beyotime Institute of Biotechnology) according to the manufacturer's instructions. A BCA kit was used to assess protein concentration (Beyotime Institute of Biotechnology). A total of $10 \mu \mathrm{g}$ protein was loaded per lane. After being separated by $10-12 \%$ sodium dodecyl sulfate-polyacrylamide gel electrophoresis, proteins were transferred to polyvinylidene fluoride membranes (EMD Millipore, Billerica, MA, USA). The membranes were blocked with $5 \%$ non-fat milk, followed by incubation with the following primary antibodies overnight at $4^{\circ} \mathrm{C}$ : anti-MCT4 (dilution 1:1,000; cat. no. 22787-1-AP; ProteinTech), anti- $\alpha$ tubulin antibody (dilution 1:1,000; cat. no. ab7291; Abcam, Cambridge, UK), anti-p-Ser308-Akt (dilution 1:1,000; cat. no. ab38449; Abcam), anti-Bax (dilution 1:1,000; cat. no. 50559-2-1g; ProteinTech Group Inc.), anti-Bcl-2 (dilution 1:1,000; cat. no. 2872; Cell Signaling Technology Inc., Danvers, MA, USA), anti-cytochrome $c$ (dilution 1:1,000; cat. no. 4272; Cell Signaling Technology), anti-cleaved caspase-3 (dilution 1:1,000; cat. no. 9661; Cell Signaling Technology), anti-caspase-3 (dilution 1:1,000; cat. no. 9662; Cell Signaling Technology). After incubation with horseradish peroxidase-labeled secondary antibodies (dilution 1:5,000; cat. no. ZB-2301; Zhongshan Golden Bridge 
Table I. The association of the clinicopathological characteristics of ESCC with MCT4 expression in FFPE tissues.

\begin{tabular}{|c|c|c|c|}
\hline \multirow[b]{2}{*}{$\begin{array}{l}\text { Clinicopathological } \\
\text { features }\end{array}$} & \multicolumn{2}{|c|}{$\begin{array}{c}\text { MCT4 } \\
\text { overexpression }\end{array}$} & \multirow[b]{2}{*}{ P-value } \\
\hline & $\begin{array}{l}\text { Low } \\
(\mathrm{n}=47)\end{array}$ & $\begin{array}{l}\text { High } \\
(\mathrm{n}=63)\end{array}$ & \\
\hline Age (years) & & & 0.680 \\
\hline$<65$ & 22 & 27 & \\
\hline$\geq 65$ & 25 & 36 & \\
\hline Sex & & & 0.060 \\
\hline Female & 12 & 27 & \\
\hline Male & 35 & 36 & \\
\hline Smoking & & & 0.798 \\
\hline No & 19 & 27 & \\
\hline Yes & 28 & 36 & \\
\hline Drinking & & & 0.116 \\
\hline No & 19 & 35 & \\
\hline Yes & 28 & 28 & \\
\hline Differentiation & & & 0.864 \\
\hline Well & 21 & 26 & \\
\hline Moderate & 12 & 19 & \\
\hline Poor & 14 & 18 & \\
\hline T stage & & & $0.001^{\mathrm{a}}$ \\
\hline $\mathrm{T} 1$ & 7 & 5 & \\
\hline $\mathrm{T} 2$ & 26 & 16 & \\
\hline $\mathrm{T} 3$ & 11 & 25 & \\
\hline $\mathrm{T} 4$ & 3 & 17 & \\
\hline $\mathrm{N}$ stage & & & $0.020^{\mathrm{a}}$ \\
\hline No & 26 & 18 & \\
\hline N1 & 5 & 19 & \\
\hline $\mathrm{N} 2$ & 11 & 18 & \\
\hline N3 & 5 & 8 & \\
\hline TNM stage & & & $0.042^{\mathrm{a}}$ \\
\hline I & 14 & 9 & \\
\hline II & 16 & 17 & \\
\hline III & 17 & 37 & \\
\hline
\end{tabular}

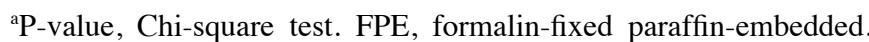
ESCC, esophageal squamous cell carcinoma; FFPE, formalin fixed and paraffin-embedded; TNM, formalin fixed and paraffin-embedded.

Biotechnology), the membranes were finally developed with Immobilon Western Chemiluminescent HRP Substrate kit (EMD Millipore). ImageJ 1.44 software was used for analyzing the protein bands (US National Institutes of Health, Bethesda, MD, USA).

Cell Counting Kit-8 (CCK-8) assay. After transfection, logarithmic growth phase cells from the sh-NC and sh-MCT4 groups were seeded at a cell density of 5,000 cells/well in 96-well plates and cultured for 0,24, 48 and $72 \mathrm{~h}$. After each of these intervals, $10 \mu \mathrm{l}$ CCK-8 solution in $100 \mu \mathrm{l}$ fresh medium

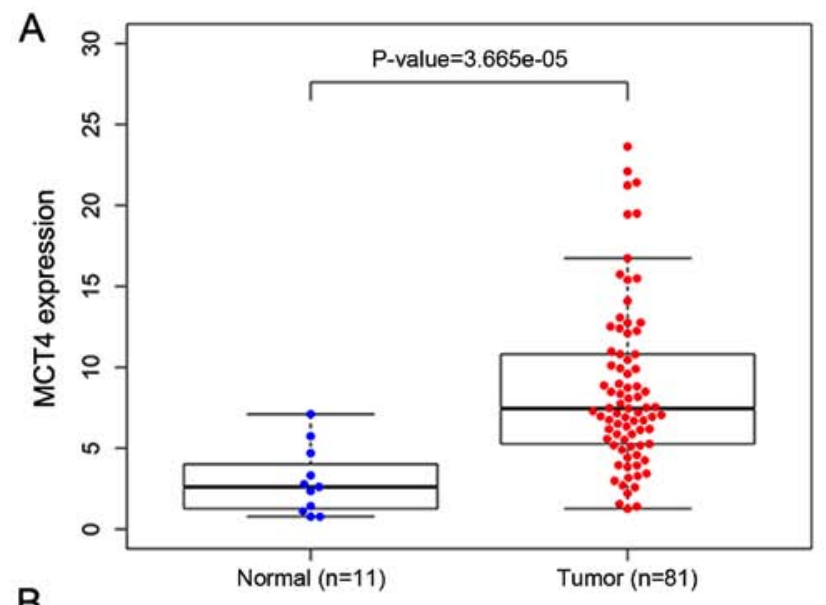

B
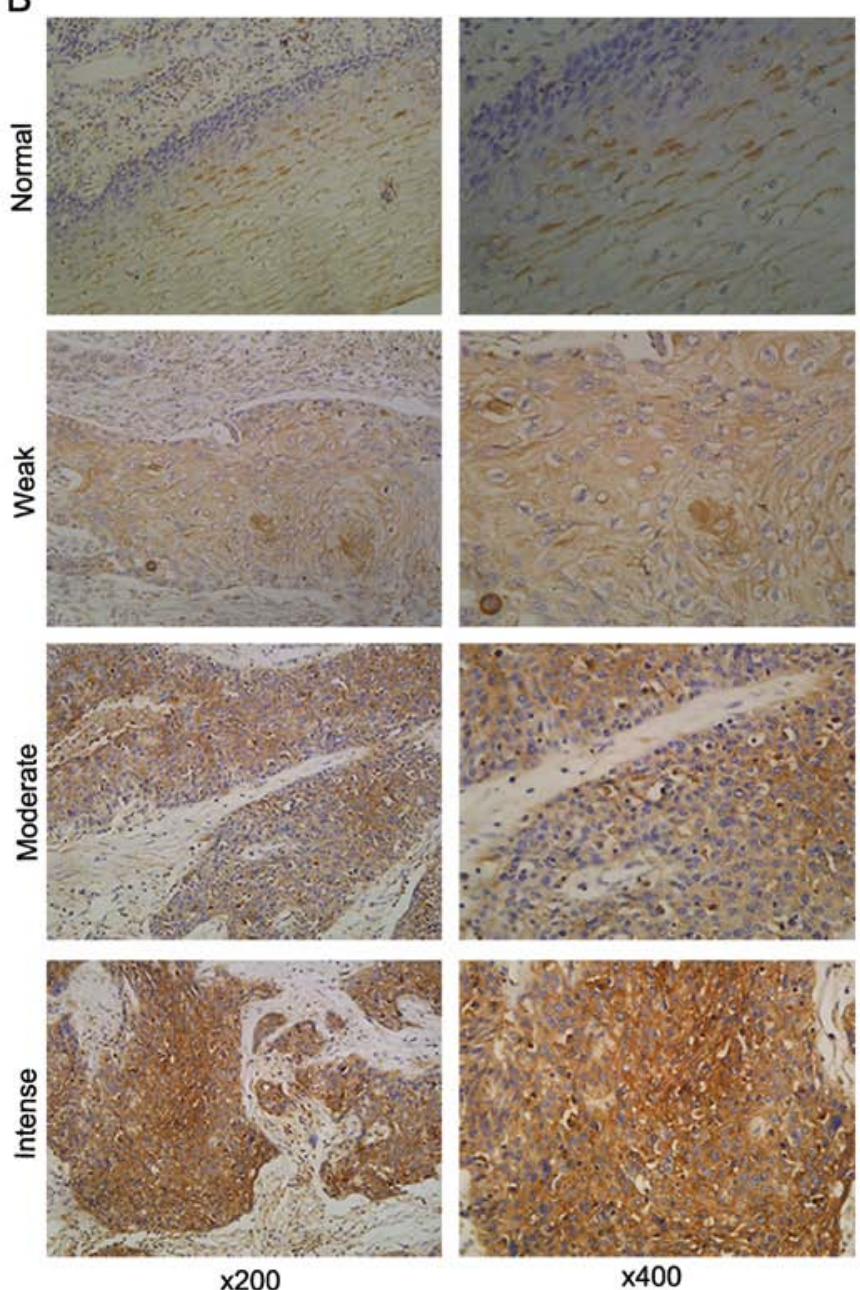

Figure 1. (A) MCT4 mRNA expression data in ESCC acquired from TCGA. (B) Representative immunostaining of MCT4 in ESCC tissues: From top to bottom, in order, are as follows: Paracancerous normal tissues, and weak, moderate and intense staining of cancerous tissues. MCT4, monocarboxylate transporter 4; ESCC, esophageal squamous cell carcinoma.

was added into each well, followed by additional incubation for $2 \mathrm{~h}$. The absorbance at $450 \mathrm{~nm}$ was measured using a Varioskan Flash spectrophotometer (Thermo Fisher Scientific, Inc.).

Clonogenic assay. Transfected cells were trypsinized and suspended in a single cell suspension. A total of 800 cells/well were seeded and cultured for 2 weeks. Ethanol was used as a 

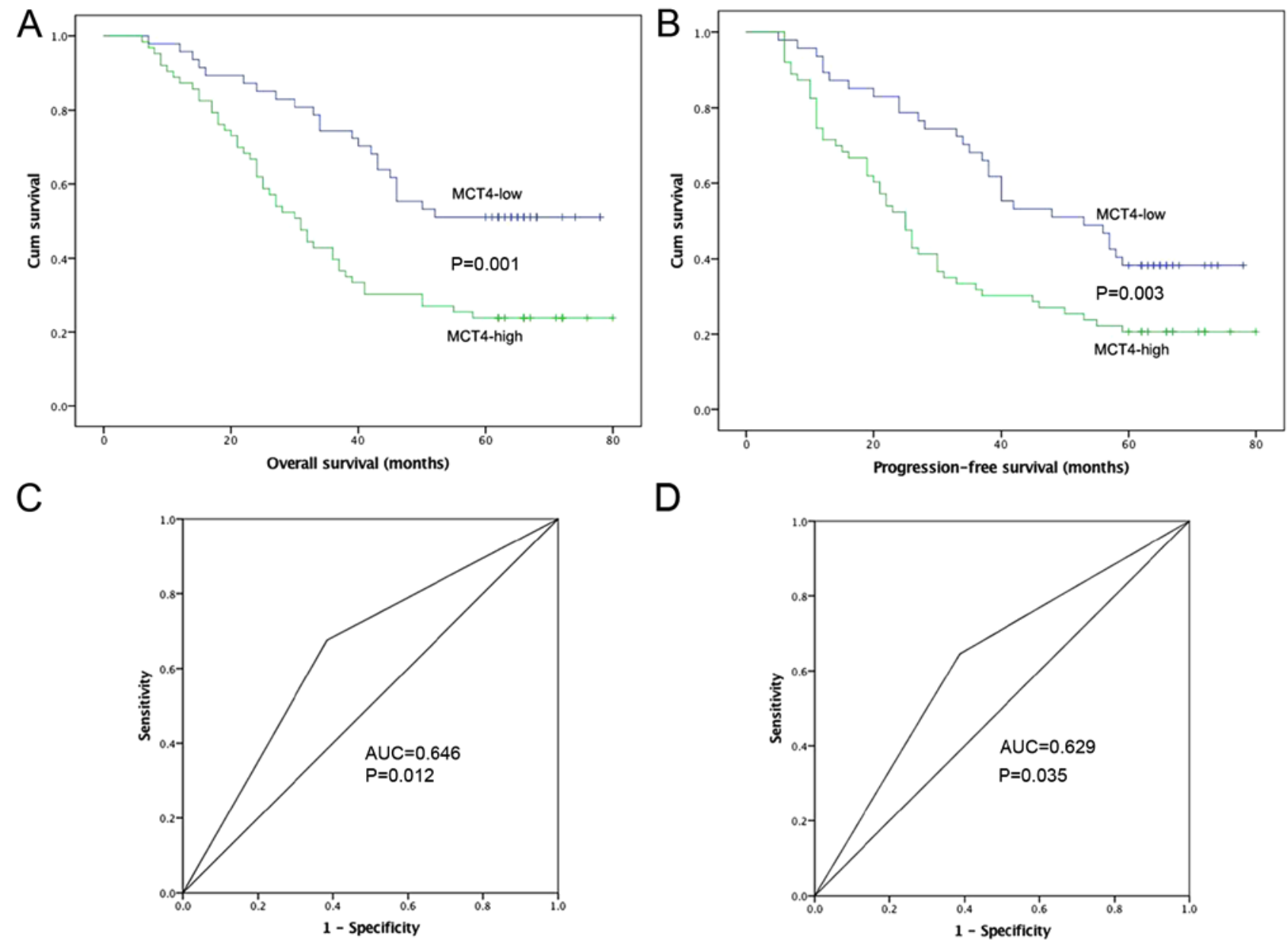

D

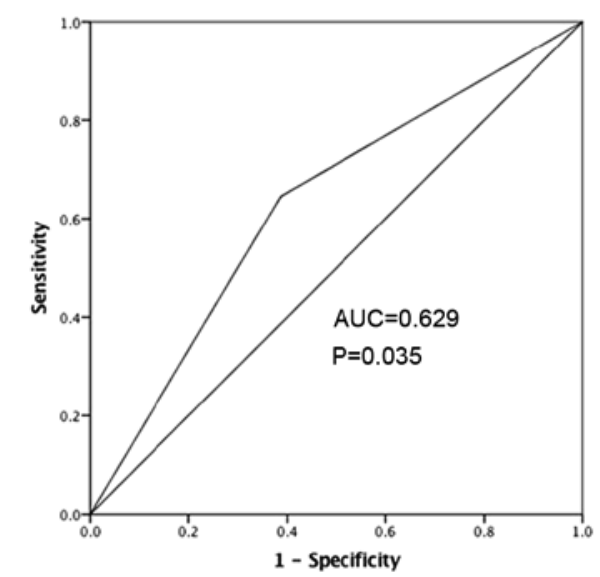

Figure 2. Kaplan-Meier curves and ROC curve analyses of MCT4 in ESCC tissues. (A) Kaplan-Meier curve for OS. (B) Kaplan-Meier curve for PFS (C) Receiver operating curve for OS. (D) Receiver operating curve for PFS. MCT4, monocarboxylate transporter 4; ESCC, esophageal squamous cell carcinoma; OS, overall survival; PFS, progression-free survival.

fixative and crystal violet was used as a cell dye for counting. A clone was defined as containing at least 50 cells.

Flow cytometric analysis. Cell apoptosis was evaluated using the Annexin V-APC/propidium iodide (PI) kit (BestBio, Shanghai, China). After transfection, cells were cultured for 5 days. After being trypsinized and harvested, the cells were washed with PBS twice. Cells were then resuspended in a binding buffer at $1-5 \times 10^{6}$ cells $/ \mathrm{ml}$. Subsequently, $5 \mu \mathrm{l}$ Annexin V-APC and $5 \mu \mathrm{l}$ PI were successively added to $100 \mu \mathrm{l}$ cell suspension. After incubation in the dark for $10 \mathrm{~min}, 400 \mu 1$ PBS was added to the mixture. The cells were immediately subjected to flow cytometry (Beckman Coulter, Inc., Brea, CA, USA). FlowJo 7.6 software was used for analyzing the results (FlowJo LLC, Ashland, OR, USA).

Statistical analysis. SPSS software (IBM Corp., Armonk, NY, USA) was used for statistical data analysis. The Wilcoxon rank sum test was used to compare the mRNA expression data from TCGA. The association of MCT4 expression with clinicopathological characteristics was analyzed using the Chi-square test. Kaplan-Meier analysis and log-rank test were applied to compare the differences in overall survival (OS) and progression-free survival (PFS) between the MCT4-high and MCT4-low groups. Death or disease progression were used as the outcomes for the Cox regression analysis. Multivariate regression analyses in the Cox regression model were performed to identify independent factors. Receiver operating characteristic (ROC) curve analysis was conducted for the Cox regression models and the area under the curve (AUC) value was used to indicate the forecasting ability. For the in vitro assay, the shRNA-MCT4 and control group were compared using a paired Student's t-test. A two-sided $\mathrm{P}<0.05$ was considered to indicate a statistically significant result.

\section{Results}

Relationship between clinicopathological characteristics and MCT4 expression. According to the TCGA database, MCT4 was upregulated in ESCC tissues compared to normal tissues (Fig. 1A, P<0.001). MCT4 was detected on the cell membranes and in the cytoplasm. Representative images of MCT4 immunostaining are shown in Fig. 1B. A total of 110 patients were studied, of whom 39 (35.5\%) were female and 71 (64.5\%) were male, with their age at the time of diagnosis ranging from 37 to 79 years (median, 61 years). The survival time of $39(35.5 \%)$ patients was more $>5$ years, while $71(64.5 \%)$ died during the follow-up period. The survival time of all patients ranged from 6-80 months (median, 39). Among the $110 \mathrm{ESCC}$ patients, 63 expressed high levels of MCT4 expression and 
Table II. Univariate analyses of prognostic variables.

\begin{tabular}{|c|c|c|c|c|c|c|}
\hline \multirow[b]{2}{*}{ Variables } & \multicolumn{3}{|c|}{ OS } & \multicolumn{3}{|c|}{ PFS } \\
\hline & P-value & HR & $95 \% \mathrm{CI}$ & P-value & HR & $95 \% \mathrm{CI}$ \\
\hline Sex (female vs. male) & 0.057 & 0.633 & $0.395-1.013$ & 0.054 & 0.642 & $0.409-1.007$ \\
\hline Age ( $<65$ vs. $\geq 65$ years) & 0.922 & 1.024 & $0.640-1.637$ & 0.612 & 0.892 & $0.573-1.388$ \\
\hline Smoking (yes vs. no) & 0.701 & 0.912 & $0.571-1.458$ & 0.693 & 0.914 & $0.586-1.427$ \\
\hline Drinking (yes vs. no) & 0.166 & 0.717 & $0.448-1.148$ & 0.256 & 0.733 & $0.496-1.205$ \\
\hline T stage & $0.001^{\mathrm{a}}$ & & & $0.001^{\mathrm{a}}$ & & \\
\hline $\mathrm{T} 1$ & & 1.000 & Ref. & & 1.000 & Ref. \\
\hline $\mathrm{T} 2$ & 0.219 & 1.949 & $0.672-5.658$ & 0.889 & 1.059 & $0.476-2.357$ \\
\hline $\mathrm{T} 3$ & $0.014^{\mathrm{a}}$ & 3.737 & $1.307-10.69$ & 0.072 & 2.057 & $0.936-4.518$ \\
\hline $\mathrm{T} 4$ & $0.001^{\mathrm{a}}$ & 5.941 & $1.988-17.75$ & $0.006^{\mathrm{a}}$ & 3.269 & $1.411-7.573$ \\
\hline $\mathrm{N}$ stage & $0.004^{\mathrm{a}}$ & & & $0.001^{\mathrm{a}}$ & & \\
\hline N0 & & 1.000 & Ref. & & 1.000 & Ref. \\
\hline N1 & 0.072 & 1.831 & $0.948-3.538$ & 0.082 & 1.747 & $0.932-3.274$ \\
\hline N2 & $0.001^{\mathrm{a}}$ & 2.723 & $1.487-4.987$ & $0.001^{\mathrm{a}}$ & 3.024 & $1.719-5.319$ \\
\hline N3 & $0.003^{\mathrm{a}}$ & 3.017 & $1.468-6.203$ & $0.003^{\mathrm{a}}$ & 2.966 & $1.461-6.021$ \\
\hline Differentiation & 0.399 & & & 0.143 & & \\
\hline Well & & 1.000 & Ref. & & 1.000 & Ref. \\
\hline Moderate & 0.427 & 1.249 & $0.722-2.159$ & 0.188 & 1.412 & $0.845-2.360$ \\
\hline Poor & 0.505 & 0.821 & $0.460-1.466$ & 0.433 & 0.800 & $0.457-1.399$ \\
\hline $\begin{array}{l}\text { MCT4 } \\
\text { (High level vs. low level) }\end{array}$ & $0.001^{\mathrm{a}}$ & 2.337 & $1.416-3.856$ & $0.004^{\mathrm{a}}$ & 1.969 & $1.242-3.122$ \\
\hline
\end{tabular}

OS, overall survival; PFS, progression-free survival; HR, hazard ratio; CI, confidence interval. ${ }^{\mathrm{a}} \mathrm{P}<0.05$ and Ref. means this item was selected as reference item.

47 expressed low levels of MCT4. The associations between clinicopathological characteristics and MCT4 expression are presented in Table I. MCT4 expression was found to be associated with $\mathrm{T}$ stage $(\mathrm{P}=0.001), \mathrm{N}$ stage $(\mathrm{P}=0.020)$ and TNM stage $(\mathrm{P}=0.042)$. No significant association was detected with clinicopathological characteristics, including sex, age, smoking habit, drinking habit and differentiation.

Prognostic value of MCT4 for ESCC patients. The OS and PFS curves of patients classified by MCT4 expression are shown in Fig. 2A and B. ROC curve analysis was conducted, and AUC values of MCT4 were $0.646(\mathrm{P}=0.012)$ and 0.629 $(\mathrm{P}=0.035)$ for $\mathrm{OS}$ and PFS, respectively (Fig. 2C and D). The effect of MCT4 expression on OS and PFS was evaluated by Kaplan-Meier analysis with the log-rank test. A decreased 5-year OS was observed in the MCT4-high group compared to the MCT4-low group ( $\mathrm{P}=0.001)$. Similarly, the MCT4-high group had a lower 5-year PFS rate $(\mathrm{P}=0.003)$.

The results of the univariate Cox regression analysis are shown in Table II. MCT4 expression $(\mathrm{P}=0.001)$, $\mathrm{T}$ stage $(\mathrm{P}=0.001)$ and $\mathrm{N}$ stage $(\mathrm{P}=0.004)$ were found to be significantly associated with OS. MCT4 expression $(\mathrm{P}=0.004)$, $\mathrm{T}$ stage $(\mathrm{P}=0.001)$ and $\mathrm{N}$ stage $(\mathrm{P}=0.001)$ were also found to be associated with PFS.

The results of the multivariate Cox regression analysis are shown in Table III. MCT4 expression $(\mathrm{P}=0.014)$, $\mathrm{T}$ stage
$(\mathrm{P}=0.038)$ and $\mathrm{N}$ stage $(\mathrm{P}=0.048)$ were found to be independent prognostic factors for ESCC patients. With respect to PFS, MCT4 expression $(\mathrm{P}=0.046)$ and $\mathrm{N}$ stage $(\mathrm{P}=0.016)$ were significantly associated with patient outcome.

Downregulation of MCT4 expression inhibits proliferation and promotes apoptosis of ESCC cells. To investigate the function of MCT4 in ESCC, shRNA was used to specifically knock down MCT4 expression in KYSE150 and Eca109 cells. RT-qPCR and western blot analyses were conducted to verify the effect of the knockdown on mRNA and protein expression levels. The expression of MCT4 mRNA (MCT4/ $\beta$-actin: $1.00 \pm 0.12$ vs. $0.55 \pm 0.10, P=0.007$ and $1.03 \pm 0.08$ vs. $0.30 \pm 0.04$, $\mathrm{P}=0.006$, for KYSE150 and Eca109 respectively; Fig. 3A) and protein (MCT4/ $\alpha$-tubulin: $1.65 \pm 0.08$ vs. $1.06 \pm 0.06$, $\mathrm{P}=0.001$ and $1.37 \pm 0.04$ vs. $0.94 \pm 0.06, \mathrm{P}<0.001$, for KYSE150 and Eca109, respectively; Fig. 3B and C) was successfully downregulated by sh-MCT4. To determine whether MCT4 affected the proliferation and survival abilities of KYSE150 and Eca109 cells, we performed CCK-8 and clonogenic assays. The OD values (Fig. 4A) of the sh-MCT4 groups were significantly decreased compared to the control sh-NC groups at 24,48 and $72 \mathrm{~h}$ for both cell lines $(\mathrm{P}<0.05)$. In comparison with the control groups, the number of clones was reduced (KYSE150, 488 \pm 21 vs. 194 $\pm 16, \mathrm{P}<0.001$; Eca109, 467 \pm 28 vs. $174 \pm 21, \mathrm{P}<0.001$; Fig. 4B and C). As shown in Fig. 4D and $\mathrm{E}$, 
Table III. Multivariate analyses of prognostic variables.

\begin{tabular}{|c|c|c|c|c|c|c|}
\hline \multirow[b]{2}{*}{ Variables } & \multicolumn{3}{|c|}{ OS } & \multicolumn{3}{|c|}{ PFS } \\
\hline & P-value & HR & $95 \% \mathrm{CI}$ & P-value & HR & $95 \% \mathrm{CI}$ \\
\hline T stage & $0.038^{\mathrm{a}}$ & & & 0.115 & & \\
\hline $\mathrm{T} 1$ & & 1.000 & Ref. & & 1.000 & Ref. \\
\hline $\mathrm{T} 2$ & 0.093 & 2.543 & $0.856-7.555$ & 0.462 & 1.364 & $0.597-3.120$ \\
\hline T3 & $0.029^{\mathrm{a}}$ & 3.285 & $1.131-9.538$ & 0.072 & 1.839 & $0.823-4.113$ \\
\hline $\mathrm{T} 4$ & $0.007^{\mathrm{a}}$ & 4.648 & $1.520-14.21$ & $0.028^{\mathrm{a}}$ & 2.626 & $1.108-6.224$ \\
\hline $\mathrm{N}$ stage & $0.048^{\mathrm{a}}$ & & & $0.016^{\mathrm{a}}$ & & \\
\hline No & & 1.000 & Ref. & & 1.000 & Ref. \\
\hline N1 & 0.479 & 1.220 & $0.605-2.458$ & 0.549 & 1.227 & $0.628-2.397$ \\
\hline N2 & $0.011^{\mathrm{a}}$ & 2.335 & $1.219-4.474$ & $0.003^{\mathrm{a}}$ & 2.517 & $1.376-4.606$ \\
\hline N3 & 0.070 & 1.730 & $0.791-3.788$ & 0.107 & 1.878 & $0.873-4.041$ \\
\hline $\begin{array}{l}\text { MCT4 } \\
\text { (High level vs. low level) }\end{array}$ & $0.014^{\mathrm{a}}$ & 2.022 & $1.153-3.479$ & $0.046^{\mathrm{a}}$ & 1.677 & $1.010-2.784$ \\
\hline
\end{tabular}

OS, overall survival; PFS, progression-free survival; HR, hazard ratio; CI, confidence interval. ${ }^{\mathrm{P}}<0.05$ and Ref. means this item was selected as reference item.

A

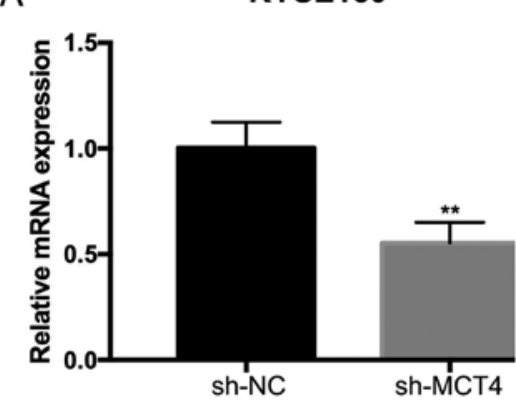

B

C

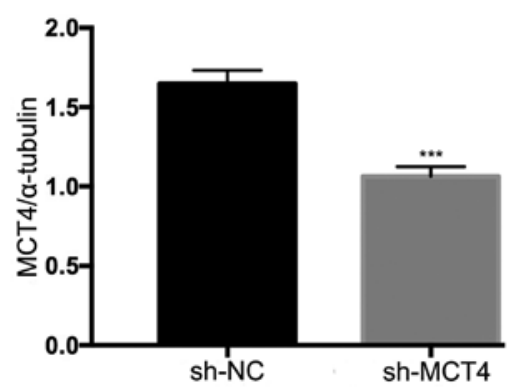

Eca109
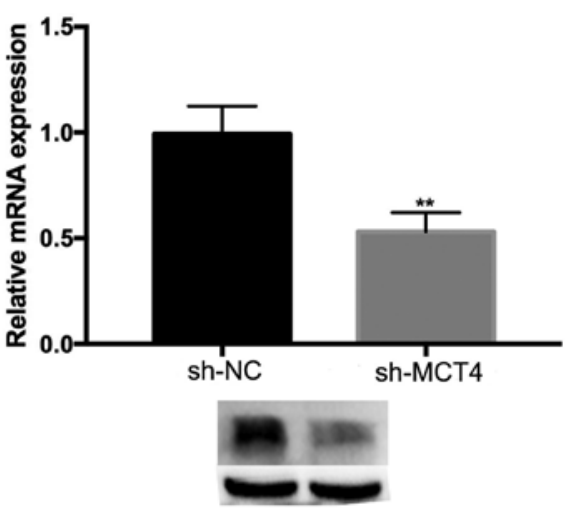

Eca109

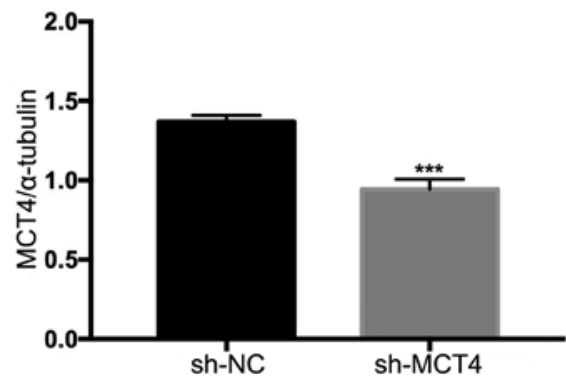

Figure 3. Downregulation effect of sh-MCT4. (A) sh-MCT4 decreased mRNA expression of MCT4. (B and C) Expression of MCT4 protein in KYSE150 and Eca109 was downregulated after sh-MCT4 transfection. ${ }^{* *} \mathrm{P}<0.01,{ }^{* * *} \mathrm{P}<0.001$, by Student's t-test. MCT4, monocarboxylate transporter 4.

5 days after shRNA was transfected, the apoptosis rates $(\%)$ of the sh-MCT4 groups were increased (KYSE150, 14.23 \pm 1.99 vs. $2.07 \pm 1.23, \mathrm{P}<0.001$; Eca109, $13.52 \pm 1.75$ vs. $2.94 \pm 1.53$, $\mathrm{P}=0.002)$.

MCT4 knockdown inhibits activation of Akt and increases Bax/Bcl-2 ratio, cytochrome c release and caspase-3 cleavage.
To explore the molecular mechanisms underlying inhibited proliferation and elevated apoptosis by loss of MCT4, key intracellular molecules of proliferation and apoptosis were examined by western blotting, including p-Akt, Bax, Bcl-2, cytochrome $c$, caspase-3 and cleaved caspase-3 (Fig. 5A). As shown in Fig. 5A, phosphorylation of Akt was decreased in sh-MCT4 groups compared to sh-NC groups (p-Akt/ $\alpha$-tubulin, 
A

B

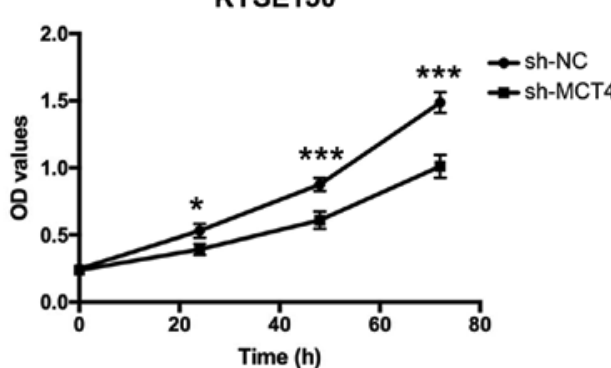

shRNA-MCT4
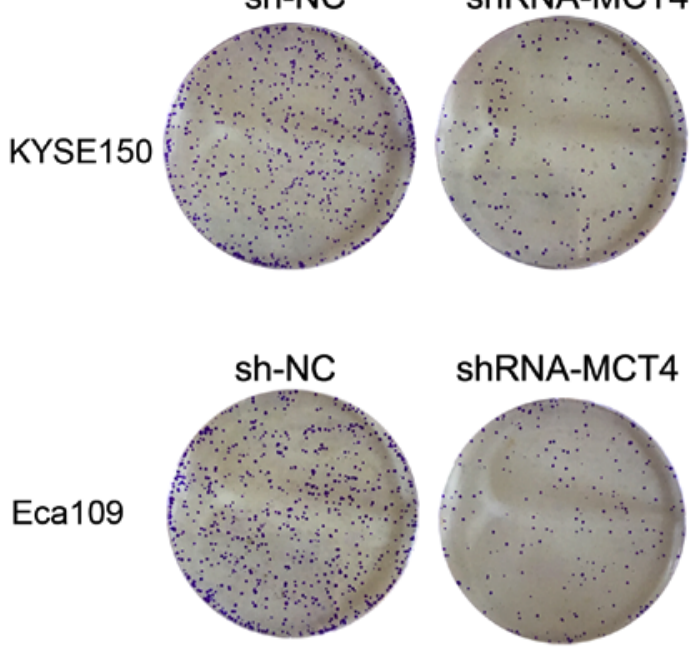

D

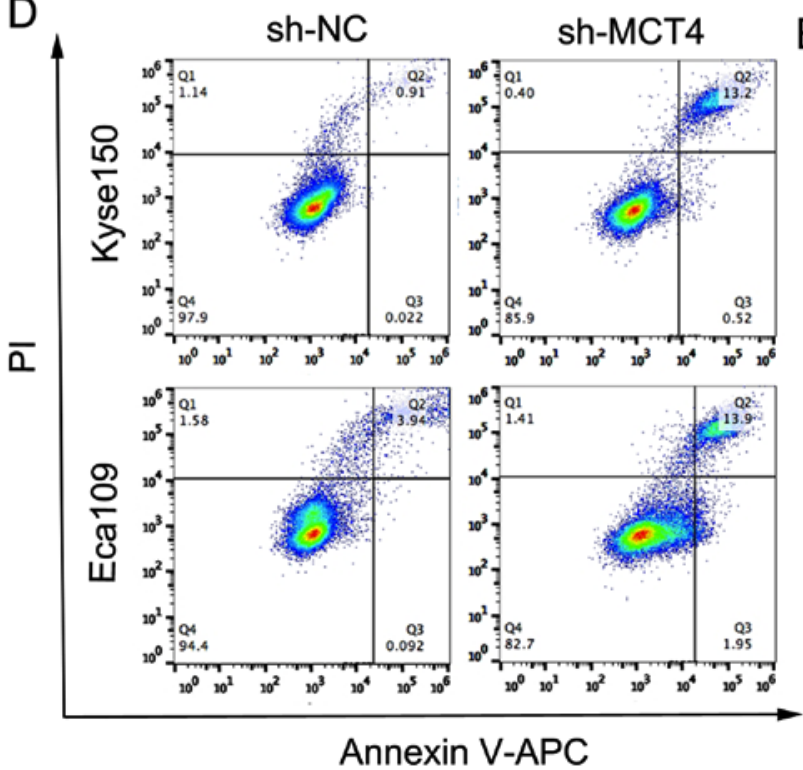

shRNA-MCT4

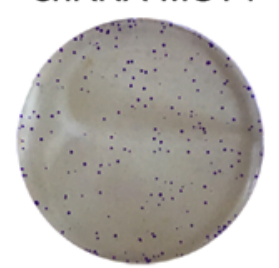

sh-MCT4

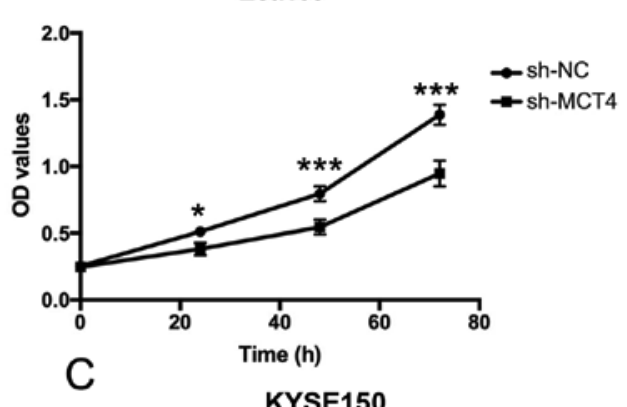

KYSE150

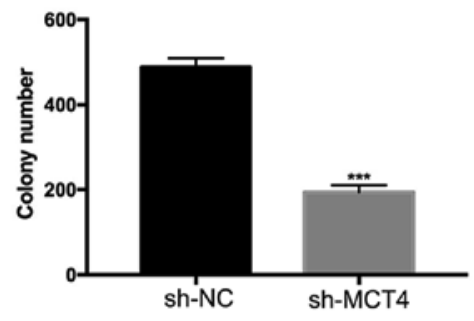

Eca109

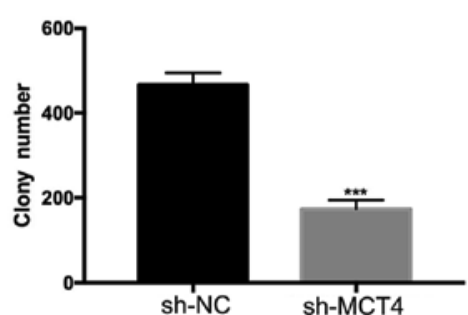

E

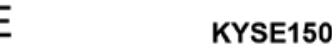

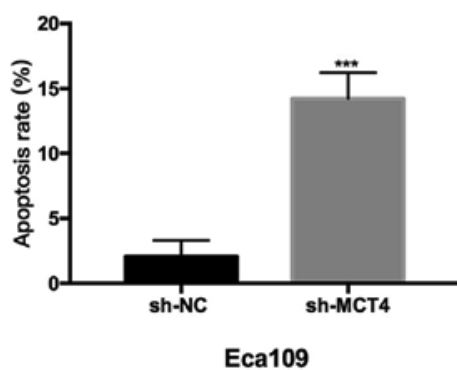

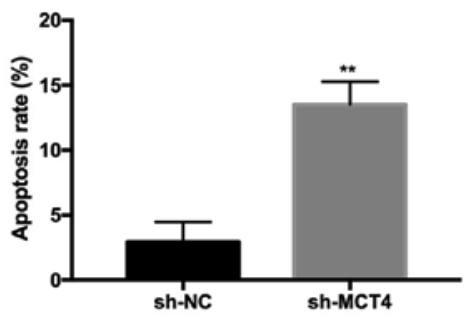

Figure 4. MCT4 regulates the proliferation ability of ESCC cells in vitro. (A) CCK-8 assays in KYSE150 and Eca109 cells. (B and C) Clonogenic assay in KYSE150 and Eca109 cells. (D and E) Flow cytometric analysis of apoptosis. ${ }^{*} \mathrm{P}<0.05,{ }^{* *} \mathrm{P}<0.01,{ }^{* * *} \mathrm{P}<0.001$, by Student's t-test. MCT4, monocarboxylate transporter 4; ESCC, esophageal squamous cell carcinoma; CCK-8, Cell Counting Kit-8.

$0.43 \pm 0.07$ vs. $0.73 \pm 0.05, P=0.004$ and $0.30 \pm 0.05$ vs. $0.54 \pm 0.07$ $\mathrm{P}=0.007$, for KYSE150 and Eca109 respectively; Fig. 5B). MCT4 knockdown upregulated Bax expression (Bax/ $\alpha$ tubulin, $1.64 \pm 0.15$ vs. $0.70 \pm 0.11, \mathrm{P}<0.001$ and $1.10 \pm 0.09$ vs. $0.67 \pm 0.11, \mathrm{P}=0.007$, for KYSE150 and Eca109 respectively; Fig. 5C) and downregulated Bcl-2 expression (Bcl-2/ $\alpha$-tubulin, $0.43 \pm 0.06$ vs. $0.72 \pm 0.10, \mathrm{P}=0.013$ and $0.35 \pm 0.03$ vs. $0.82 \pm 0.03$, $\mathrm{P}<0.001$, for KYSE150 and Eca109 respectively; Fig. 5D), thus increasing the Bax/Bcl-2 ratio (KYSE150, 3.86 \pm 0.27 vs.
$0.97 \pm 0.11, \mathrm{P}<0.001 ;$ Eca109, 3.14 \pm 0.24 vs. $0.81 \pm 0.12, \mathrm{P}<0.001$; Fig. 5E). Cytoplasmic cytochrome $c$ release was increased with loss of MCT4 (cytoplasmic cytochrome $c / \alpha$-tubulin, $0.69 \pm 0.09$ vs. $0.31 \pm 0.09, \mathrm{P}=0.006$ and $0.89 \pm 0.08$ vs. $0.39 \pm 0.08, \mathrm{P}=0.002$, for KYSE150 and Eca109 respectively; Fig. 5F). Additionally, MCT4 knockdown promoted caspase-3 cleavage (cleaved caspase-3/ $\alpha$-tubulin, $0.55 \pm 0.06$ vs. $0.20 \pm 0.05, P=0.002$ and $0.46 \pm 0.05$ vs. $0.13 \pm 0.03 \mathrm{P}<0.0001$, for KYSE150 and Eca109 respectively; Fig. 5G). 
A

D

$\mathrm{F}$

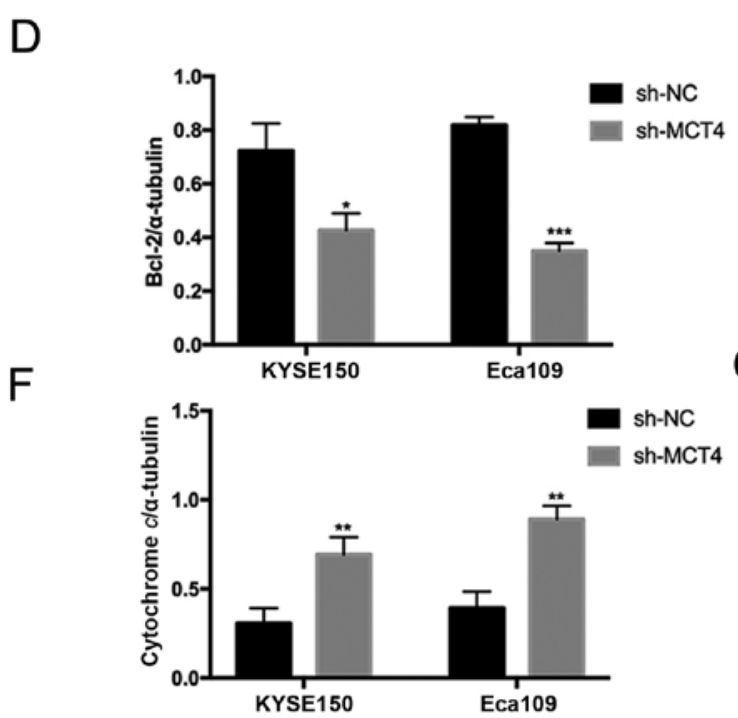

$\mathrm{E}$
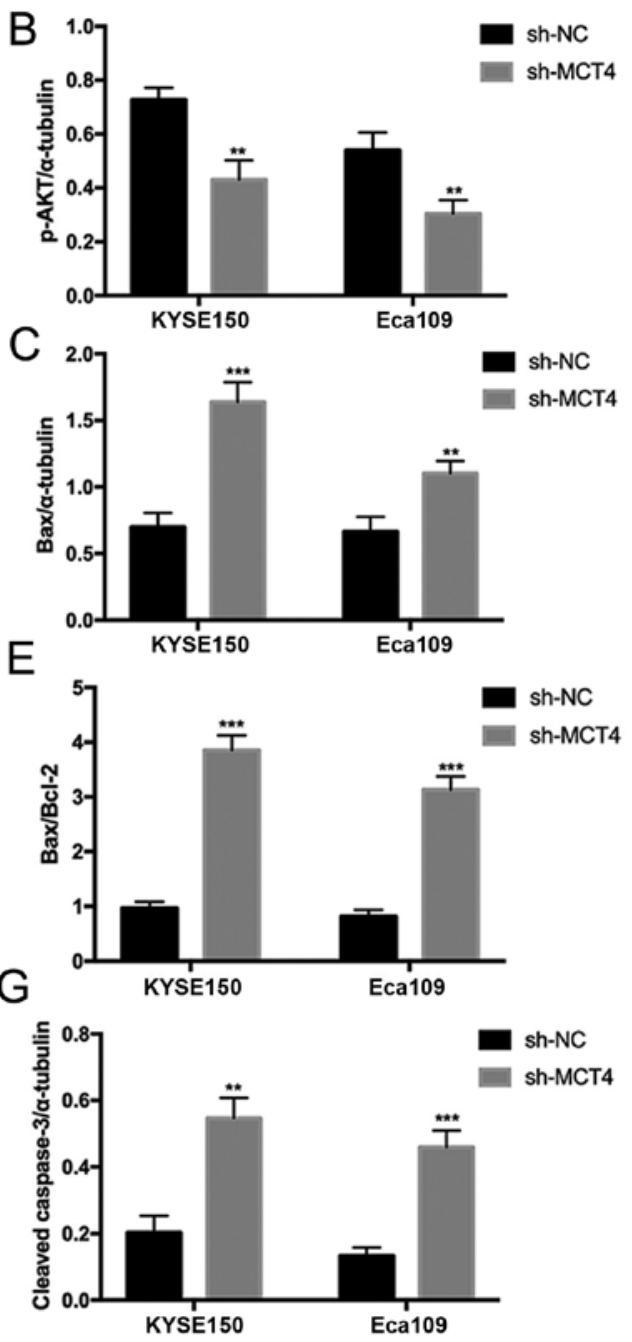

Figure 5. Antiproliferative and pro-apoptotic effects of MCT4 knockdown on ESCC cells by decreasing the activation of Akt, Bax/Bcl-2 ratio, cytochrome $c$ release and caspase-3 cleavage. (A) Immunoblots and immunoblotting analysis of (B) p-Akt, (C-E) Bax and Bcl-2, (F) cytoplasmic cytochrome $c$ and (G) cleaved caspase-3. ${ }^{*} \mathrm{P}<0.05,{ }^{* *} \mathrm{P}<0.01,{ }^{* * *} \mathrm{P}<0.001$, by Student's t-test. MCT4, monocarboxylate transporter 4; ESCC, esophageal squamous cell carcinoma.

\section{Discussion}

According to the 'Warburg effect', cancer cells exhibit a greater dependency on the glycolytic pathway for ATP generation (20). Rapid glucose uptake and lactate secretion are features of the 'Warburg effect' that contribute to sustained ATP production and provide biosynthetic intermediates in many types of cancer (21). The widespread clinical application of 18 fluorodeoxyglucose (FdG) positron-emission tomography (PET) has demonstrated that the glycolytic phenotype exists in most cancer types (22). The main function of MCT4 is secretion of lactate and protons from highly glycolytic cells in order to stabilize the intracellular environment (23).

According to data acquired from the TCGA database, MCT4 is expressed at higher levels in ESCC cancerous tissue compared with normal tissue. It is possible that MCT4 may act as an oncogene in ESCC, as it does in other types of cancer $(11,12,14-19)$ and it could be a potential prognostic factor for ESCC. Analysis of the associations between patient clinicopathological characteristics and MCT4 expression revealed that MCT4 expression was significantly associated with $\mathrm{T}$ stage, $\mathrm{N}$ stage and TNM stage. Kaplan-Meier analysis indicated that patients with lower MCT4 expression had a higher survival rate. ROC-AUC analysis indicates the degree of concordance between expected and observed ordering of the data. The AUC values of MCT4 were statistically significant for prediction of OS and PFS. Univariate and multivariate Cox regression analyses demonstrated that MCT4 was an independent prognostic factor for ESCC patients. Furthermore, in vitro assays indicated that knockdown of MCT4 by shRNA-MCT4 decreased the proliferation and increased the apoptosis rates of ESCC cells. Accordingly, MCT4 could potentially be used as a prognostic biomarker for survival of ESCC patients.

The altered metabolism of cancer cells is controlled by oncogene signaling and mutated metabolic enzymes (24). Cancers with invasion and metastasis tendency and rapid development in a hypoxic microenvironment usually exhibit upregulated expression of MCT4, mediated by hypoxia-inducible factor 1 (HIF-1) and CD147 (25). In addition, oncogene signaling pathways can also be influenced by the metabolites generated from reciprocally reprogrammed metabolism and such crosstalk may sustain the progression of cancer and its resistance to therapeutic agents (26). Based upon the finding 
that loss of MCT4 inhibited proliferation and promoted apoptosis of ESCC cells, it is proposed that MCT4 downregulation leads to the alteration of gene expression associated with proliferation and apoptosis. Akt signaling plays a crucial role in various cellular processes, including cell proliferation, apoptosis, intracellular transport, glucose metabolism, genetic transcription and cell migration (27).

In the present study, we found that phosphorylation of Akt was inhibited by MCT4 knockdown in two ESCC cell lines. This result indicated that MCT4 expression was associated with activation of Akt signaling. Furthermore, increased Bax/ Bcl-2 ratio, cytochrome $c$ release and caspase-3 cleavage were observed in MCT4 knockdown ESCC cells. Previous evidence suggests that pro- and anti-apoptotic activities are tightly regulated by the $\mathrm{Bcl}-2$ family (28). These proteins regulate cell apoptosis by controlling the release of cytochrome $c$, through adjusting the permeability of the mitochondrial membrane (29). Bax is inserted into the mitochondrial membrane and facilitates the release of cytochrome $c$ into the cytoplasm, while Bcl-2 impedes cytochrome $c$ release (30). Cytochrome $c$ promotes the intrinsic pathway of apoptosis, which is activated after its combination with deoxyadenosine triphosphate (dATP) and apoptotic protease-activating factor (Apaf1) to form an apoptosome (31). Then, the apoptosome provides a platform to gather the initiating caspases and eventually activated caspase- 3 , which is a general executioner of apoptosis, by cleaving numerous substrates inside the cell (32). The current results indicated that MCT4 knockdown had an apoptosis-inducing effect on ESCC cells via the mitochondria pathway. A potential mechanism of proliferative inhibition and apoptotic activation is that MCT4 is not only essential for the hyper-glycolytic phenotype, but also for the acid-resistant phenotype by eliminating newly generated lactate, thus allowing cells to continuously convert pyruvate to lactate, conduct glycolysis and resist acid-induced apoptosis or necrosis (33). MCT4 silencing leads to intracellular acidosis and reduction of ATP production, together with partial reversion of the 'Warburg effect' in clear cell renal cell carcinoma (ccRCC) cell lines (34). MCT4 knockdown may also cause lactate to accumulate, lessen the ATP production of glycolysis and eventually result in decreased proliferation and increased apoptosis of ESCC cells. Although the mechanism remains unclear, MCT4 could be an attractive target for ESCC therapy based upon our findings.

In conclusion, the results of this study indicated that MCT4 is a novel biomarker for predicting the outcomes of ESCC patients. Downregulation of MCT4 decreased proliferation and increased apoptosis of ESCC cells by reducing activation of Akt and initiating the mitochondrial apoptosis pathway. Targeting MCT4 may serve as an effective treatment for ESCC patients.

\section{Acknowledgements}

Not applicable.

\section{Funding}

The present study was supported by a grant from the National Natural Science Foundation of China (grant no. 81671785).

\section{Availability of data and materials}

The analyzed data sets are available from the corresponding author upon reasonable request.

\section{Authors' contributions}

JY and $\mathrm{BC}$ designed the study. $\mathrm{BC}, \mathrm{XC}$ and $\mathrm{YL}$ generated the majority of the data. $\mathrm{XH}$ provided the follow-up data. $\mathrm{BC}$ was the major writer of the manuscript. All authors read and approved the manuscript and agree to be accountable for all aspects of the research in ensuring that the accuracy or integrity of any part of the work are appropriately investigated and resolved.

\section{Ethics approval and consent to participate}

The present study was approved by the Ethics Committee of Qilu Hospital (Jinan, China) and was carried out in compliance with the 1964 Helsinki declaration and its later amendments. As the present study was a retrospective study, the requirement to obtain informed consent was waived. All patient data were treated in accordance with the privacy regulations of our committee.

\section{Patient consent for publication}

Not applicable.

\section{Competing interests}

The authors declare that they have no competing interests.

\section{References}

1. Napier KJ, Scheerer M and Misra S: Esophageal cancer: A Review of epidemiology, pathogenesis, staging workup and treatment modalities. World J Gastrointest Oncol 6: 112-120, 2014.

2. Abnet CC, Arnold M and Wei WQ: Epidemiology of esophageal squamous cell carcinoma. Gastroenterology 154: 360-373, 2018.

3. Mandard AM, Hainaut P and Hollstein M: Genetic steps in the development of squamous cell carcinoma of the esophagus. Mutat Res 462: 335-342, 2000.

4. Ohashi S, Miyamoto S, Kikuchi O, Goto T, Amanuma Y and Muto M: Recent advances from basic and clinical studies of esophageal squamous cell carcinoma. Gastroenterology 149: 1700-1715, 2015

5. Halestrap AP and Price NT: The proton-linked monocarboxylate transporter (MCT) family: Structure, function and regulation. Biochem J 343: 281-299, 1999.

6. Halestrap AP and Wilson MC: The monocarboxylate transporter family - role and regulation. IUBMB Life 64: 109-119, 2012.

7. Juel C and Halestrap AP: Lactate transport in skeletal muscle - role and regulation of the monocarboxylate transporter. J Physiol 517: 633-642, 1999.

8. Bonen A: The expression of lactate transporters (MCT1 and MCT4) in heart and muscle. Eur J Appl Physiol 86: 6-11, 2001.

9. Bergersen L, Rafiki A and Ottersen OP: Immunogold cytochemistry identifies specialized membrane domains for monocarboxylate transport in the central nervous system. Neurochem Res 27: 89-96, 2002.

10. Lunt SY and Vander Heiden MG: Aerobic glycolysis: Meeting the metabolic requirements of cell proliferation. Annu Rev Cell Dev Biol 27: 441-464, 2011.

11. Doyen J, Trastour C, Ettore F, Peyrottes I, Toussant N, Gal J, Ilc K, Roux D, Parks SK, Ferrero JM, et al: Expression of the hypoxiainducible monocarboxylate transporter MCT4 is increased in triple negative breast cancer and correlates independently with clinical outcome. Biochem Biophys Res Commun 451: 54-61, 2014. 
12. Kwon JE, Jung WH and Koo JS: The expression of metabolismrelated proteins in phyllodes tumors. Tumour Biol 34: 115-124, 2013.

13. Witkiewicz AK, Whitaker-Menezes D, Dasgupta A, Philp NJ, Lin Z, Gandara R, Sneddon S, Martinez-Outschoorn UE, Sotgia F and Lisanti MP: Using the 'reverse Warburg effect' to identify high-risk breast cancer patients: Stromal MCT4 predicts poor clinical outcome in triple-negative breast cancers. Cell Cycle 11: 1108-1117, 2012.

14. Gao HJ, Zhao MC, Zhang YJ, Zhou DS, Xu L, Li GB, Chen MS and Liu J: Monocarboxylate transporter 4 predicts poor prognosis in hepatocellular carcinoma and is associated with cell proliferation and migration. J Cancer Res Clin Oncol 141: 1151-1162, 2015.

15. Ohno A, Yorita K, Haruyama Y, Kondo K, Kato A, Ohtomo T, Kawaguchi M, Marutuska K, Chijiiwa K and Kataoka H: Aberrant expression of monocarboxylate transporter 4 in tumour cells predicts an unfavourable outcome in patients with hepatocellular carcinoma. Liver Int 34: 942-952, 2014.

16. Baek G, Tse YF, Hu Z, Cox D, Buboltz N, McCue P, Yeo CJ White MA, DeBerardinis RJ, Knudsen ES, et al: MCT4 defines a glycolytic subtype of pancreatic cancer with poor prognosis and unique metabolic dependencies. Cell Reports 9: 2233-2249, 2014.

17. Nakayama Y, Torigoe T, Inoue $Y$, Minagawa N, Izumi H, Kohno K and Yamaguchi K: Prognostic significance of monocarboxylate transporter 4 expression in patients with colorectal cancer. Exp Ther Med 3: 25-30, 2012.

18. Zhu J, Wu YN, Zhang W, Zhang XM, Ding X, Li HQ, Geng M, Xie ZQ and Wu HM: Monocarboxylate transporter 4 facilitates cell proliferation and migration and is associated with poor prognosis in oral squamous cell carcinoma patients. PLoS One 9: e87904, 2014.

19. Zhao Z, Han F, He Y, Yang S, Hua L, Wu J and Zhan W: Stromalepithelial metabolic coupling in gastric cancer: Stromal MCT4 and mitochondrial TOMM20 as poor prognostic factors. Eur J Surg Oncol 40: 1361-1368, 2014.

20. Vander Heiden MG, Cantley LC and Thompson CB: Understanding the Warburg effect: The metabolic requirements of cell proliferation. Science 324: 1029-1033, 2009.

21. Shlomi T, Benyamini T, Gottlieb E, Sharan R and Ruppin E: Genome-scale metabolic modeling elucidates the role of proliferative adaptation in causing the Warburg effect. PLOS Comput Biol 7: e1002018, 2011.
22. Gatenby RA and Gillies RJ: Why do cancers have high aerobic glycolysis? Nat Rev Cancer 4: 891-899, 2004.

23. Dimmer KS, Friedrich B, Lang F, Deitmer JW and Bröer S: The low-affinity monocarboxylate transporter MCT4 is adapted to the export of lactate in highly glycolytic cells. Biochem J 350: 219-227, 2000.

24. Cairns RA, Harris IS and Mak TW: Regulation of cancer cell metabolism. Nat Rev Cancer 11: 85-95, 2011.

25. Parks SK, Chiche J and Pouyssegur J: $\mathrm{pH}$ control mechanisms of tumor survival and growth. J Cell Physiol 226: 299-308, 2011.

26. Blanker MH, Norg RJ and van der Heide WK: A new approach to patients with lower urinary tract symptoms. Br J Gen Pract 62: 344-345, 2012

27. Manning BD and Cantley LC: AKT/PKB signaling: Navigating downstream. Cell 129: 1261-1274, 2007.

28. Youle RJ and Strasser A: The BCL-2 protein family: Opposing activities that mediate cell death. Nat Rev Mol Cell Biol 9: 47-59, 2008.

29. Cory S, Huang DC and Adams JM: The Bcl-2 family: Roles in cell survival and oncogenesis. Oncogene 22: 8590-8607, 2003.

30. Oltvai ZN, Milliman CL and Korsmeyer SJ: Bcl-2 heterodimerizes in vivo with a conserved homolog, Bax, that accelerates programmed cell death. Cell 74: 609-619, 1993.

31. Riedl SJ and Salvesen GS: The apoptosome: Signalling platform of cell death. Nat Rev Mol Cell Biol 8: 405-413, 2007.

32. Fan TJ, Han LH, Cong RS and Liang J: Caspase family proteases and apoptosis. Acta Biochim Biophys Sin (Shanghai) 37: 719-727, 2005.

33. Hsieh FY and Lavori PW: Sample-size calculations for the Cox proportional hazards regression model with nonbinary covariates. Control Clin Trials 21: 552-560, 2000.

34. Gerlinger M, Santos CR, Spencer-Dene B, Martinez P, Endesfelder D, Burrell RA, Vetter M, Jiang M, Saunders RE, Kelly G, et al: Genome-wide RNA interference analysis of renal carcinoma survival regulators identifies MCT4 as a Warburg effect metabolic target. J Pathol 227: 146-156, 2012. 